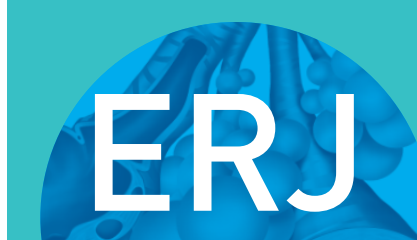

open research

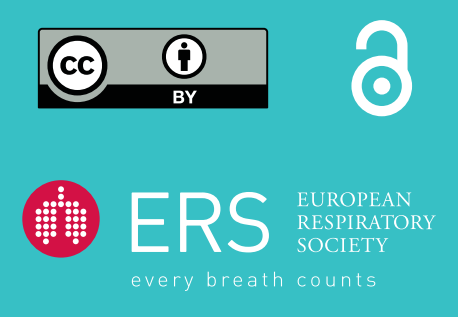

\section{“Trans-basement membrane migration of eosinophils induced by LPS-stimulated neutrophils from human peripheral blood in vitro" Fuyumi Nishihara, Kazuyuki Nakagome, Takehito Kobayashi, Toru Noguchi, Ryuichiro Araki, Yoshitaka Uchida, Tomoyuki Soma and Makoto Nagata. ERJ Open Res 2015; 1: 00003-2015.}

This article was originally published with incorrect p-values in figures 2 and 6 . The revised figures are shown below and have been corrected in the article itself. The authors apologise for this error.

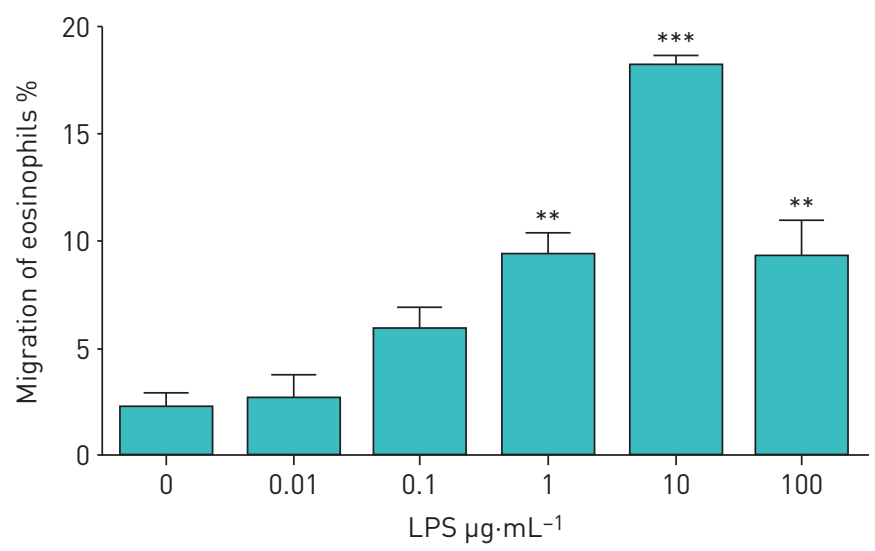

FIGURE 2 Dose-dependent effect of lipopolysaccharide (LPS) on neutrophil-induced trans-basement membrane migration of eosinophils in healthy volunteers. Neutrophils $\left(2 \times 10^{4}\right.$ cells $)$ were stimulated with various concentrations of LPS $\left(0.01-100 \mu \mathrm{g} \cdot \mathrm{mL}^{-1}\right)$ and then placed into the lower compartment. Eosinophils $\left(1 \times 10^{5}\right.$ cells $)$ were added to the upper compartment of a chamber with a Matrigel-coated Transwell insert. After 120 min of incubation, migrated eosinophils in the lower chamber were measured by eosinophil peroxidase assays $(n=4)$. Data are presented as mean \pm SEM. ${ }^{* *}$ : $p<0.01$ versus spontaneous migration $\left(0 \mu \mathrm{g} \cdot \mathrm{mL}^{-1}\right.$ LPS) by Tukey test; ${ }^{* * *}: \mathrm{p}<0.001$ versus spontaneous migration $\left(0 \mu \mathrm{g} \cdot \mathrm{mL}^{-1} \mathrm{LPS}\right)$ by Tukey test.

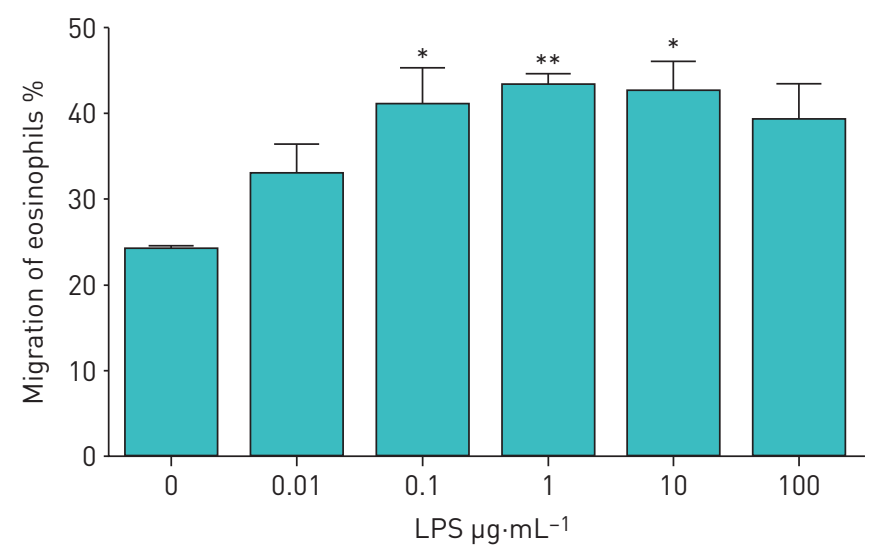

FIGURE 6 Dose-dependent effect of lipopolysaccharide (LPS) on neutrophil-induced trans-basement membrane migration of eosinophils in severe asthmatics. Neutrophils $\left(2 \times 10^{4}\right.$ cells $)$ from severe asthmatics were stimulated with various concentrations of LPS $\left(0.01-100 \mu \mathrm{g} \cdot \mathrm{mL}^{-1}\right)$, and then placed into the lower compartment of a chamber with a Matrigel-coated Transwell insert. Eosinophils $\left(1 \times 10^{5}\right.$ cells $)$ from severe asthmatics were added to the upper compartment. After $120 \mathrm{~min}$ of incubation, migrated eosinophils in the lower chamber were measured by eosinophil peroxidase assays $(n=4)$. Data are presented as mean \pm SEM. *: $p<0.05$ versus spontaneous migration $\left(0 \mu \mathrm{g} \cdot \mathrm{mL}^{-1}\right.$ LPS) by Tukey test; ${ }^{* *}$ : $p<0.01$ versus spontaneous migration $\left(0 \mu \mathrm{g} \cdot \mathrm{mL}^{-1} \mathrm{LPS}\right)$ by Tukey test.

ERJ Open Res 2017; 3: 00003-2015-AU | DOI: 10.1183/23120541.50003-2017 | Copyright (CERS 2017 This article is open access and distributed under the terms of the Creative Commons Attribution NonCommercial Licence 4.0 